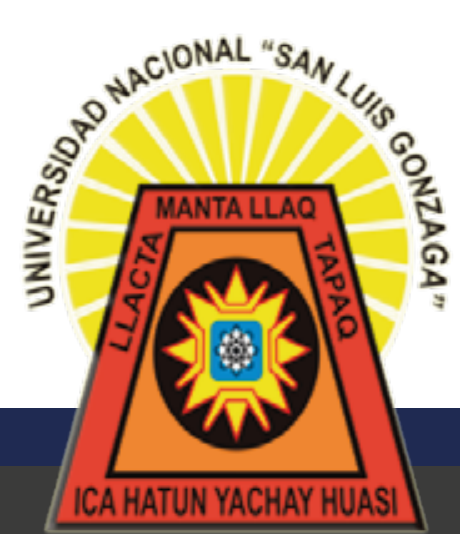

p-ISSN 2223-2893

e-ISSN 2225-6989

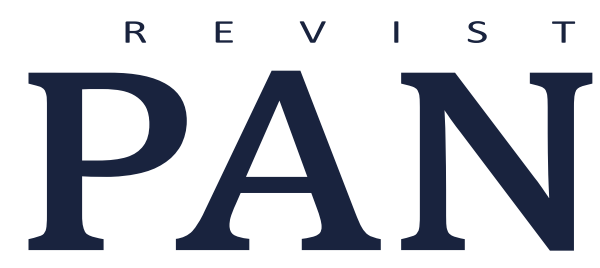

UNIVERSIDAD NACIONAL SAN LUIS GONZAGA. ICA, PERÚ

FACULTAD DE MEDICINA HUMANA "DANIEL ALCIDES CARRIÓN"

ARTÍCULO ORIGINAL:

VOLUMEN 10 NÚMERO 2 PUBLICACION CUATRIMESTRAL MAYO - AGOSTO 2021

\title{
NIVEL DE CONOCIMIENTO EN LA PREVENCION DE LAS ENFERMEDADES TRANSMISIBLES POR AEDES AEGYPTI EN EL DISTRITO SAN JOSÉ DE LOS MOLINOS DICIEMBRE 2018.
}

LEVEL OF KNOWLEDGE IN THE PREVENTION OF TRANSMISSIBLE DISEASES BY AEGYPTI AEDES IN THE SAN JOSÉ DE LOS MOLINOS DISTRICT DECEMBER 2018.

\section{AUTORES:}

TAIPE MARQUINA JERSHON ESTEBAN

PRETELL AYULO BERTHA HORTENCIA

INDEXADA EN:

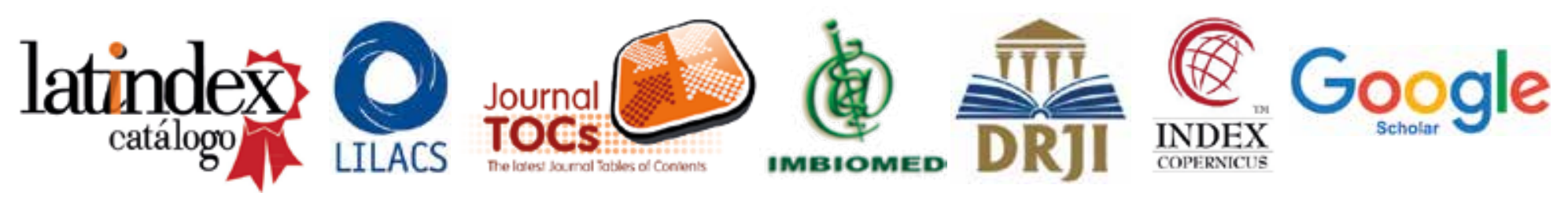

REVISTAS.UNICA.EDU.PE 


\title{
NIVEL DE CONOCIMIENTO EN LA PREVENCION DE LAS ENFERMEDADES TRANSMISIBLES POR AEDES AEGYPTI EN EL DISTRITO SAN JOSÉ DE LOS MOLINOS DICIEMBRE 2018.
}

\section{LEVEL OF KNOWLEDGE IN THE PREVENTION OF TRANSMISSIBLE DISEASES BY AEGYPTI AEDES IN THE SAN JOSÉ DE LOS MOLINOS DISTRICT DECEMBER 2018.}

\author{
Taipe-Marquina Jershon Esteban ${ }^{1, a}$, Pretell-Ayulo Bertha Hortencia ${ }^{1, a, b}$ \\ 1. Facultad de Medicina Humana. Universidad Nacional San Luis \\ Gonzaga. Ica, Perú. \\ a. Médico cirujano \\ b. Médico anatomopatólogo
}

DOI: https://doi.org/10.35563/rmp.v10i2.428

Correspondencia:

Taipe Marquina Jershon

Esteban

Dirección: Urb Las Flores Avellanos B-8.

Correo electrónico:

yersontm@hotmaill.com

Celular: 989272393

Contribuciones de autoría: TMJE y PABH: Participaron en la concepción y diseño del manuscrito, recolección, análisis e interpretación de los datos, redacción y revisión crítica del contenido del manuscrito y aprobación final del artículo.

Conflicto de intereses: no existen conflictos de intereses del autor o autores de orden económico, institucional, laboral o personal.

Financiamiento:

Autofinanciado.

\section{Cómo citar:}

Taype-Marquina $\mathrm{J}$

Pretell-Ayulo B. Nivel de conocimiento en la prevención de las enfermedades transmisibles por Aedes aegypti en el distrito San José de los Molinos diciembre 2018. Rev méd panacea 2021;10(2): 80-83. DOI: https://doi.org/10.35563 /rmp.v10i2.428

Recibido: 24 - 05 - 2021 Aceptado: 08 - $06-2021$ Publicado: 12 - 08 - 2021

\section{RESUMEN}

Objetivo: Determinar el nivel de conocimiento en la prevención de las enfermedades transmisibles por Aedes Aegypti en el Distrito de Los Molinos diciembre 2018. Materiales y métodos: Estudio de tipo observacional, transversal, no experimental. Realizada en 362 pobladores del distrito de los Molinos. Resultados: El nivel de conocimiento para dengue es de $52,76 \%$ alto, el $41.44 \%$ para Zika es medio y el $48.07 \%$ es medio para chikungunya. Conclusiones: El presente estudio da como conclusiones que los niveles de conocimiento para las enfermedades transmisibles por Aedes Aegypti, no guarda relación significativa para zika y chikungunya ya que el valor esperado fue mayor a $\mathrm{P}=0.005$, mientras que para dengue si existe relación significativa ya que el valor $\mathrm{P}=0.005$ es menor.

Palabras clave: Aedes Aegypti, dengue, zika, chikungunya, nivel de conocimiento.

\section{ABSTRACT}

Objective: To determine the level of knowledge in the prevention of communicable diseases by Aedes Aegypti in the District of Los Molinos December 2018. Material and methods: Observational, cross-sectional, non-experimental study. Made in 362 residents of the district of Los Molinos. Results: The level of knowledge for dengue is $52.76 \%$ high, $41.44 \%$ for Zika is medium and $48.07 \%$ is medium for chikungunya. Conclusions: The present study concludes that the levels of knowledge for the diseases transmissible by Aedes Aegypti, does not have a significant relationship for zika and chikungunya since the expected value was greater than $P=0.005$, while for dengue if there is a significant relationship already that the value $P=0.005$ is less.

Key words: Aedes Aegypti, dengue, zika, chikungunya, level of knowledge. 


\section{INTRODUCCIÓN}

Los mosquitos de la especie Aedes Aegypti, la cual se encuentran en todo a región del país, son aquellos vectores que logran tener la capacidad para contagiar diversas enfermedades virales tales como el dengue, la Fiebre Chikungunya, la Enfermedad por Virus Zika, etc., cuya enfermedad está afectando a las personas, esto hace que los problemas en salud aumenten (1).

En América, de acuerdo a lo registrado por la Organización Mundial de la Salud (OMS) en el año 2002, se reportaron 609 000 casos de dengue, de los cuales, 17000 fueron hemorrágicos, con una mortalidad de 225 casos (1,3\%), el doble de lo estimado para 1995 (2).

En el junio del 2014 se reportó un caso en la Isla de Pascua (Chile) y posteriormente no se volvió a detectar el virus. En el primer semestre del 2015 en el nordeste de Brasil se estuvo investigando un posible brote del virus Zika (3).

En nuestro país el número de casos de fiebre de Chikungunya aumento durante el año 2016 en comparación al año 2015, durante el año 2015 se reportaron un total de 219 casos, 173 confirmados y 46 probables, de los cuales 200 se dieron en el departamento de Tumbes y 19 en el departamento de Piura, para el año 2016 se reportaron un total de 272 casos 131 de estos fueron confirmados y 141 probables de los cuales 233 se dieron en el departamento de Tumbes, 27 en el departamento de Loreto, 1 caso en el departamento de Piura, 5 casos en el departamento de Madre de Dios, 3 en Huánuco, y 3 en San Martin, aquellos donde se reportaron casos autóctonos fueron en Loreto, Tumbes y San Martin (4).

Según el reporte de la oficina de epidemiología, hasta el 24 de abril del 2015 se reportó un brote de dengue en el distrito El Porvenir, de la provincia de Trujillo, que se inició en la semana epidemiológica 6 con presentación de casos de dengue con señales de alarma con tendencia al incremento y elevado riesgo de extensión del mismo a otros distritos con infestación del vector transmisor (5).

A partir de los años 1990 - 2014 se ha podido evidenciar un considerable incremento de casos en esta enfermedad, principalmente en los departamentos pertenecientes a la zona amazónica y norteña. Además el Instituto nacional de salud culminó monitoreo donde se estudió los efectos bioquímicos que los insecticidas comunes causaban al mosquito, y las consecuencias genéticas en su ADN (6).

Es por ello que esta investigación tiene como propósito medir los conocimientos que tiene la comunidad sobre las acciones de prevención, de esta forma, tomen conciencia de la importancia en su salud, aporten en la disminución del crecimiento de los mosquitos urbanos debido a sus estilos de vida.

\section{MATERIALES Y MÉTODOS}

La investigación que se realizó fue de tipo básica, por medio de la recolección de datos, de forma que se añade datos que profundizan cada vez los conocimientos ya existidos en la realidad, se construye a base de esto un mayor conocimiento en sus hipótesis, teorías y leyes, por eso es importante conocer los antecedentes para poder generar criterios nuevos por medio de la investigación donde se especifique la forma detallada de su estudio sus conclusiones se basaran en los hechos.

De acuerdo a su nivel fue no experimental, ya que se considero que es la búsqueda empírica y sistemática en la que el científico no posee control directo de las variables independientes, debido a que sus manifestaciones ya han ocurrido o que son inherentemente no manipulados.

El diseño de la presente investigación fue descriptivo y transversal. Descriptivo porque se apreció las situaciones y eventos de los fenómenos observados. De corte transversal, ya que se realizó en los meses de noviembre a diciembre del 2018.

En la investigación se usó dos instrumentos: Entrevista y Encuesta.

Los instrumentos que se usaron para la recolección de datos fueron las 3 hojas de encuesta con 16 preguntas de alternativas múltiples. También se empleó cuestionarios de preguntas abiertas para desarrollar la entrevista con los pobladores del distrito San José de Los Molinos. Los datos obtenidos fueron vaciados manualmente en una base de datos, posterior a ello, los resultados se plantearon en tablas y gráficos estadísticos de frecuencias relativas y absolutas para su análisis e interpretación. Para ello se empleó el software SPSS versión 22, para detallar los informes respectivos.

\section{RESULTADOS}

Participaron en el estudio 320 pacientes, la mayoría fueron de sexo masculino (51,2\%); grupo etario joven $(76,3 \%)$; grado de instrucción secundaria (72,5\%); zona de residencia urbana $(63,7 \%)$, el promedio de edad fue de 20 años. (Tabla 1$)$

Tabla 1: características sociodemográficas de los pobladores del Distrito san José de los Molinos diciembre 2018. CARACTERÍSTICA N $\quad \%$

\section{Edad}

15 A 29 años

10

2.76

30-59 años

348

96.13

$>60$ años

4

1.10

\section{Grado de instrucción}

Analfabeta

3

0.83

Primaria

92

25.56

Secundaria

148

41.11

Universitaria

119

33.06

\section{Ocupación}

Ama de casa

24.86

Comerciante

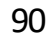

27.35

Obrero

99

38

10.50

Profesional

116

32.04

Estudiante

13

3.59

Otros

6

1.66

\section{Sexo}

Femenino

Masculino

Total

362 
En la tabla 1. Se aprecia las características sociodemográficos de los pobladores del distrito de San José de los Molinos, de la cual el grupo de edades que mayor frecuencia $96.13 \%(348)$ fue entre los 30 a 59 años, 41.11\%(148) grado de instrucción secundaria, $32.04 \%(116)$ es profesional y siendo el sexo femenino el predominante con $65.75 \%(238)$.

Tabla 2: Nivel de conocimiento de conocimientos sobre el dengue en el distrito de Los Molinos diciembre 2018.

\begin{tabular}{lcc} 
Nivel de conocimiento & $\mathbf{N}$ & $\%$ \\
Bajo & 74 & 20.44 \\
Medio & 97 & 26.80 \\
Alto & 191 & 53.76 \\
Total & 362 & 100 \\
\hline
\end{tabular}

En la tabla 2 nos muestra el nivel de conocimiento que tienen los pobladores sobre el dengue, siendo alto $53.76 \%(191)$.

Tabla 3: Nivel de conocimiento sobre el Zika en el Distrito de los Molinos diciembre 2018.

\begin{tabular}{lcc} 
Nivel de conocimiento & $\mathbf{N}$ & $\%$ \\
Bajo & 112 & 30.16 \\
Medio & 150 & 41.44 \\
Alto & 63 & 17.40 \\
Total & 362 & 100 \\
\hline
\end{tabular}

En la tabla 3 se aprecia el nivel de conocimiento que tienen los pobladores sobre el Zika, siendo medio el predominante con $41.44 \%(150)$.

Tabla 4. Nivel de conocimiento de sobre el Chikungunya en el Distrito de los Molinos diciembre 2018.

\begin{tabular}{lcc} 
Nivel de conocimiento & $\mathbf{N}$ & $\%$ \\
Bajo & 95 & 26.24 \\
Medio & 174 & 48.07 \\
Alto & 94 & 25.97 \\
Total & 362 & 100 \\
\hline
\end{tabular}

\section{DISCUSIÓN}

En la presente investigación sobre el nivel de conocimiento en la prevención de las enfermedades transmisibles por aedes aegypti en el distrito de Los Molinos diciembre 2018, se encontró que el nivel de conocimiento es medio tanto para dengue con el 52,76\%, para Zika $41,44 \%$ y Chikungunya $48 \%, 07 \%$.

Estos resultados son similares a los encontrados en el estudio de Gallegos I, Ñañez C. Nivel De Conocimiento Y Prácticas Sobre Prevención Del Dengue De Los Pobladores Atendidos En El Centro De Salud De Posope Alto - 2,016. Perú. 2,017, en la cual tiene como resultados que el $14.6 \%$ tiene conocimiento alto y el $72.9 \%$ medio y $12.5 \%$ bajo (6), dando a conocer que en Lambayeque sus resultados acerca del nivel de conocimiento para enfermedades transmisibles por Aedes Aegypti no es adecuado.
Por otro lado en este estudio de Cabrera F., Gómez de la Torre A., Alejandra Isabel Bocanegra A, Correa Borit J., Huamaní F., Urrunaga P., Elsa Casablanca E. Conocimientos, actitudes y prácticas sobre dengue en estudiantes de educación primaria en Chorrillos, Lima, Perú. 2,016, en sus resultados acerca del conocimiento es básico de dengue y $53,5 \%$ conocen como identificar la transmisión del dengue (7), lo cual hace referencia que en los estudiantes de dicha institución su conocimiento es medio al igual que nuestro estudio.

Así mismo en el estudio de Parra J., Roldán Fernández J. Conocimientos, Actitudes y Prácticas sobre el Chikungunya en la Población de la Parroquia Bartolomé Ruiz del Cantón Esmeraldas - Ecuador, 2,015, en sus resultados en cuanto al conocimiento el $36.1 \%$ tiene un conocimiento del chikungunya es regular en gran parte de la población (8).

Es por ello que Pender en su modelo de Promoción de la Salud, en el cual nos habla que los determinantes de la promoción de la salud y los estilos de vida, están divididos en factores cognitivos-perceptuales, entendidos como concepciones, creencias, ideas que tienen las personas sobre la salud, las cuales inducen a conductas o comportamientos determinados (9), en relación con los resultados obtenidos podemos afirmar que la percepción que tienen los pobladores del dengue, Zika y chikungunya es medio, por ello se puede esperar o inducir que las conductas con relación a estas enfermedades no serán favorecedoras para lograr una buena promoción de la salud respecto a este tema.

\section{CONCLUSIONES}

El nivel de conocimientos sobre el Dengue en el Distrito de Los Molinos de los meses de noviembre a diciembre del 2018, es alto con el $52.76 \%$, conocimientos sobre el zika es medio con el $41,44 \%$ y el nivel de conocimientos sobre Chikungunya es medio con el $48,07 \%$. 


\section{REFERENCIAS BIBLIOGRÁFICAS}

1. Dirección General De Epidemiologia. Alerta Epidemiológica Ante Incremento De Riesgo Estacional De La Transmisión De Dengue En El País. Lima-Peru; 2014. Disponible en: http://www.dge.gob.pe/portal/docs/alertas/2014/AE001.pdf

2. Nuñez Muñoz J. Conocimientos y actitudes frente a la enfermedad del dengue en madres de familia que asisten al Centro de Salud "La Tinguiña", Junio de 2017. [Tesis para optar título profesional]. Ica: Universidad Autónoma de Ica, Facultad de Ciencias de la Salud- Enfermería; 2017. Disponible en: http://repositorio.autonomadeica.edu.pe/handle/autonoma deica/155

3. Dra. Cristina Fernández Barrantes.virus zika en Centro de Información de Medicamentos Servicio de Farmacia del Hospital San Juan de Dios.2015.disponible en : http://www.medigraphic.com/pdfs/revcliescmed/ucr-2015/ ucr154m.pdf

4. Martínez Ríos N. Conocimientos sobre Fiebre de Chikungunya en pobladores del distrito de Pátapo y Pucalá, Lambayeque 2015. [Tesis para optar título profesional] Lambayeque: Universidad San Martin de Porres, Facultad de Medicina Humana; 2015. Disponible en:

http://www.repositorioacademico.usmp.edu.pe/bitstream/u smp/2541/1/MARTINEZ_NF.pdf

5. Rodríguez A. Conocimientos, Actitudes Y Prácticas Sobre La Prevención Del Dengue En Los Hogares De Río Seco, Sector 3 - El Porvenir - Trujillo 2016. [Tesis para optar título profesional]. Trujillo: Universidad Cesar Vallejo, Facultad de Enfermería; 2016. Disponible en: http://repositorio.ucv.edu.pe/bitstream/handle/UCV/778/ro driguez_ta.pdf?sequence $=1$

6. Gallegos I, Ñañez C. Nivel De Conocimiento Y Prácticas Sobre Prevención Del Dengue De Los Pobladores Atendidos En El Centro De Salud De Posope Alto - 2016. Lambayeque: Universidad Señor de Sipán; Facultad De Ciencias De La Salud - Escuela Académica Profesional De Enfermería; 2016. Disponible en:

http://repositorio.uss.edu.pe/xmlui/handle/uss/3341

7. Cabrera F., Gómez de la Torre A., Alejandra Isabel Bocanegra A, Correa Borit J., Huamaní F., Urrunaga P., Elsa Casablanca E. Conocimientos, actitudes y prácticas sobre dengue en estudiantes de educación primaria en Chorrillos, Lima, Perú. Lima: Universidad Peruana de Ciencias Aplicadas, Escuela de Medicina; 2016.

8. Paladines Vélez N., Quizhpi Jiménez M., Paladines Vélez G., Parra Parra J., Roldán Fernández J. Conocimientos, Actitudes y Prácticas sobre el Chikungunya en la Población de la Parroquia Bartolomé Ruiz del Cantón Esmeraldas - Ecuador, 2015. [Trabajo de investigación]. Ecuador; Esmeraldas. 2015.

9. Marriner A., Raile M. Modelos y Teorías en Enfermería. 5ta edición. Madrid - España. Editorial Elsevier España S.A.; 2010 p. $324-326$.

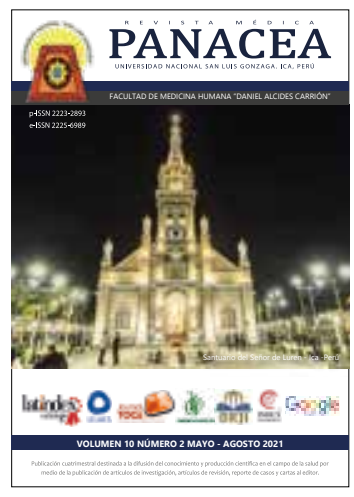

